\title{
The Impact of Vaccination on Influenza-Related Respiratory Failure and Mortality in Hospitalized Elderly Patients Over the 2013-2014 Season
}

\author{
Manish Joshi ${ }^{*}, 1$, Deepak Chandra ${ }^{2}$, Penchala Mittadodla ${ }^{1}$ and Thaddeus Bartter ${ }^{1}$ \\ ${ }^{I}$ Pulmonary and Critical Care Division, University of Arkansas for Medical Sciences, Little Rock, AR, Central Arkansas \\ Veterans Healthcare System, Little Rock, AR 72205, USA \\ ${ }^{2}$ Department of Medicine, University of Arkansas for Medical Sciences, Little Rock, AR, Central Arkansas Veterans \\ Healthcare System, Little Rock, AR 72205, USA
}

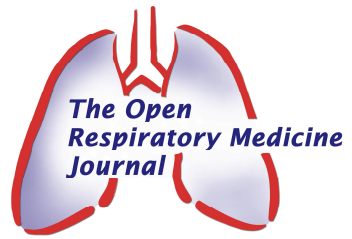

\begin{abstract}
Background: Seasonal Influenza ("the flu") is a respiratory illness caused by influenza viruses. Yearly influenza vaccination is considered to be protective against illness and/or severity of illness and is recommended by CDC for all individuals $>6$ months of age. However, the effectiveness of influenza vaccine in older individuals has come under question.

Objectives: To describe the clinical characteristics and treatment outcomes of patients admitted to an academic tertiary care Veterans Administration hospital with influenza during the 2013-2014 influenza season and determine the impact, if any, of prior influenza vaccination upon patient outcomes.

Methods: Medical electronic records were searched for all patients admitted to the Little Rock Veterans Administration Hospital with proven influenza during the 2013-2014 influenza season. Cohorts of vaccinated and non-vaccinated patients were then compared to determine the impact of prior influenza vaccination upon respiratory-failure and mortality.

Results: Seventy patients met selection criteria. Mean age was 66 years. Sixty-four (91\%) patients had at least one underlying co-morbid condition; these conditions included COPD, congestive heart failure, diabetes, and cancer. 60/70 $(85 \%)$ tested positive for Influenza A, and 43 tested positive for H1N1. Oseltamivir was initiated in 55 (78\%) patients. Forty-four percent of the patients had been vaccinated. When separated by vaccination status, those who had been vaccinated had higher rates of ICU admission, need for mechanical or non-invasive ventilation, and mortality. All but mortality reached statistical significance.

Conclusion: The data suggest that there was no protective effect from prior vaccination in preventing hospital admission, respiratory failure, and mortality in this population of older men admitted to the hospital with influenza.
\end{abstract}

Keywords: Influenza, vaccine.

\section{INTRODUCTION}

Influenza ("the flu") is a respiratory illness caused by influenza viruses. It affects a large number of Americans on a yearly basis, causing a spectrum of illness ranging from self-limiting disease to disease severe enough to warrant hospital admission [1,2]. The United States Centers for Disease Control and Prevention (CDC) states that flu vaccination can reduce flu-related morbidity and mortality, especially in the elderly with underlying medical conditions [2-4] but the true efficacy of the vaccine has come under question $[5,6]$. The objectives of this study were to assess the respiratory-failure and mortality in an elderly patient population admitted to an academic tertiary care center in the United States for influenza and to determine whether prior vaccination had an impact upon severity of illness.

*Address correspondence to this author at the Pulmonary and Critical Care Division, University of Arkansas for Medical Sciences, Little Rock, AR, Central Arkansas Veterans Healthcare System, $4300 \mathrm{~W} 7^{\text {th }}$ Street, 5C 144, Little Rock, AR 72205, USA; Tel: 1-501-257-5786; Fax: 1-501-686-7893; E-mail: Manish.Joshi@va.gov

\section{METHODS}

A retrospective chart review was conducted at the Central Arkansas Veterans Healthcare System (CAVHS) after obtaining the approval of the CAVHS Institutional Review Board (599896-2). We requested a waiver of informed consent and was approved by the IRB as this is a retrospective chart review which involves minimal risk to the participants. All data reported in this study are anonymous. As part of standard patient care, all patients evaluated at CAVHS with a clinical suspicion of the "flu" are tested for influenza at the CAVHS lab using the influenza A/B panel. This includes naso-pharyngeal swab testing for influenza rapid antigen and Influenza A reverse transcriptasepolymerase chain reaction (RT-PCR) for all who have either negative rapid test results or test positive for flu A. All labproven influenza (rapid and/or RT-PCR test positive) patients admitted to the hospital from October 1, 2013 to February 28, 2014 were included in this study.

Electronic medical records of infected patients were queried to collect data on age, gender, influenza test results, 
length of stay, intensive care unit (ICU) stay, need for positive pressure ventilatory support (PPV), treatments including oseltamivir and antibiotics, and clinical outcomes. We also obtained information on influenza vaccination status; date, month, route of administration, and type of influenza vaccination were directly obtained from the electronic medical records. Study participants were considered vaccinated if they had received $\geq 1$ dose of any seasonal influenza vaccine $\geq 14$ days before onset of illness.

\section{Statistical Analysis}

Statistical analysis was conducted using SAS 9.3 version (SAS Institute, Cary, NC). All reported p-values were twotailed and calculated with statistical significance set at $\mathrm{p} \leq 0.05$. Chi-square as well as Fisher exact test was used, as appropriate to compare categorical variables. Independent group t-testing was used to compare continuous variables.

\section{RESULTS}

A total of 70 patients were admitted to the Little Rock VA with lab-proven Influenza over the study interval. The patients ranged from 47 to 91 years of age, but were largely older; mean and median ages were 66 and 65, respectively. Only one patient (aged 47) was younger than 50 years of age, $11(16 \%)$ were in the 50-60 year age range, and 58 $(83 \%)$ were over 60 . Only 3 of the 70 patients were women. Sixty of the seventy influenza patients $(86 \%)$ were positive for influenza A and the remaining $10(14 \%)$ for influenza $B$. The majority of influenza A disease was due to H1N1 infection; 54 of the 60 influenza A patients had been subtyped using RT-PCR and 43 of the 54 were positive for H1N1. Sixty-four of the 70 patients $(91 \%)$ had at least one underlying co-morbid condition; these conditions included COPD, congestive heart failure, diabetes, and cancer. Fiftyfive $(79 \%)$ of the 70 patients were treated with oseltamivir, and $51(73 \%)$ were treated with antibiotics (with a large number receiving both). Nineteen patients were admitted to the ICU for respiratory failure. Seventeen patients $(24 \%)$ received positive pressure ventilatory support, with a roughly even $(11 \% / 13 \%)$ distribution between non-invasive ventilation and mechanical ventilation. Four patients died, for an overall mortality of $6 \%$.

Breakdown by vaccination status is presented in Table $\mathbf{1 .}$ The vaccinated and non-vaccinated groups were roughly equal; 31 (44\%) had received a trivalent inactivated influenza vaccine $\geq 14$ days before onset of illness, and 39 $(56 \%)$ had not received any prior vaccination. Most of these non-vaccinated individuals refused the vaccine (as documented in their medical records). There was no statistically significant difference between the groups with respect to the percentage of patients with comorbidities, the percent with infiltrates on Chest X-ray, and the percent treated with oseltamivir.

\section{ICU Admissions and Positive Pressure Requirements by Vaccination Status}

The rate of influenza vaccination in patients requiring ICU care was higher than in patients admitted to general
Table 1. Clinical characteristics of 70 influenza patients. They are divided into vaccinated $(n=31,44 \%)$ and unvaccinated $(n=39,56 \%)$ group for comaprision. There was no statistically significant difference between the groups except in a) age $\geq 60$ years; b) ICU admission; and c) use of positive pressure ventilation (marked with*).

\begin{tabular}{|l|c|c|}
\hline Patient Parameters & $\begin{array}{c}\text { Vaccinated } \\
\mathbf{N}=\mathbf{3 1}\end{array}$ & $\begin{array}{c}\text { Unvaccinated } \\
\mathbf{N}=\mathbf{3 9}\end{array}$ \\
\hline \hline Age Range & & \\
$<50$ years & 0 & $1(3 \%)$ \\
$50-59$ years & $1(3 \%)$ & $10(25 \%)$ \\
$\geq 60$ years* & $30(97 \%)$ & $28(72 \%)$ \\
\hline Gender & $31(100 \%)$ & $36(92 \%)$ \\
Male & 0 & $3(8 \%)$ \\
Female & $31(100 \%)$ & $33(85 \%)$ \\
\hline Comorbidities (any) & $18(58 \%)$ & $18(46 \%)$ \\
COPD & $11(35 \%)$ & $9(23 \%)$ \\
Diabetes Mellitus & $7(23 \%)$ & $2(5 \%)$ \\
CHF & $6(19 \%)$ & $8(21 \%)$ \\
Immunocompromised & & \\
\hline Type of Influenza & $25(81 \%)$ & $35(90 \%)$ \\
Influenza A & $16(52 \%)$ & $27(69 \%)$ \\
H1N1 & $6(19 \%)$ & $4(10 \%)$ \\
\hline Influenza B & $11(35 \%)$ & $14(36 \%)$ \\
\hline Infiltrates on CXR & $24(77 \%)$ & $31(79 \%)$ \\
\hline Received Tamiflu & $24(77 \%)$ & $27(69 \%)$ \\
\hline Antibiotic therapy & 6 & 4 \\
\hline Median length of stay (days) & $12(39 \%)$ & $7(18 \%)$ \\
\hline Admitted to ICU* & $6(19 \%)$ & $5(13 \%)$ \\
\hline PPV* & $7(23 \%)$ & $1(3 \%)$ \\
Mechanical ventilation & & $38(97 \%)$ \\
Non-invasive ventilation & $(90 \%)$ & \\
\hline Outcome & & $(3 \%)$ \\
Recovery & & \\
Death & & \\
\hline
\end{tabular}

medical floor; $63.2 \%(12 / 19)$ of patients requiring ICU admission had been vaccinated, compared with only $37.3 \%$ $(19 / 51)$ of patients treated on general medical floor $(\mathrm{p}=$ 0.05 ) (Fig. 1). Similarly, 76.5\% (13/17) of patients who required positive pressure ventilation had been vaccinated, compared with only $33.9 \%$ (18/53) of patients who did not require positive pressure ventilation $(\mathrm{p}=0.004)$ (Fig. 2).

Both age and COPD, the most prevalent comorbidity, were evaluated to determine whether they were independent predictors of ICU admission or the need for ventilatory support for respiratory failure. No significant correlations were found. Mean ages for those admitted to ICU versus those treated on the medical floor were 65.8 and 65.7, respectively $(p=0.969)$ (Fig. 3). Mean ages for those requiring PPV and those not requiring PPV during the hospital stay were 67.6 and 65.1 years, respectively 


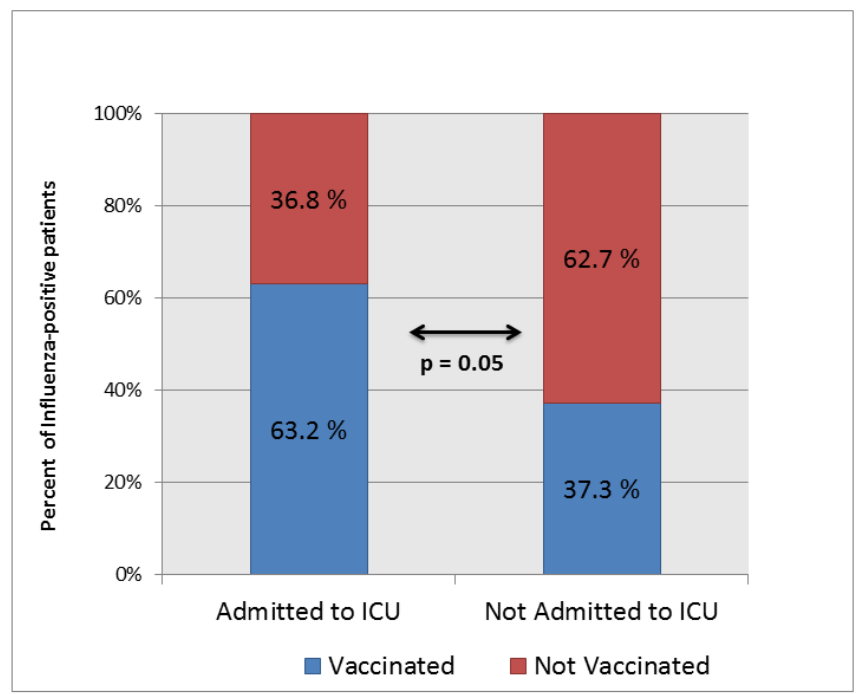

Fig. (1). $100 \%$ Stacked Column chart showing high rate of influenza vaccination in ICU patients as compared to those treated in non-ICU settings

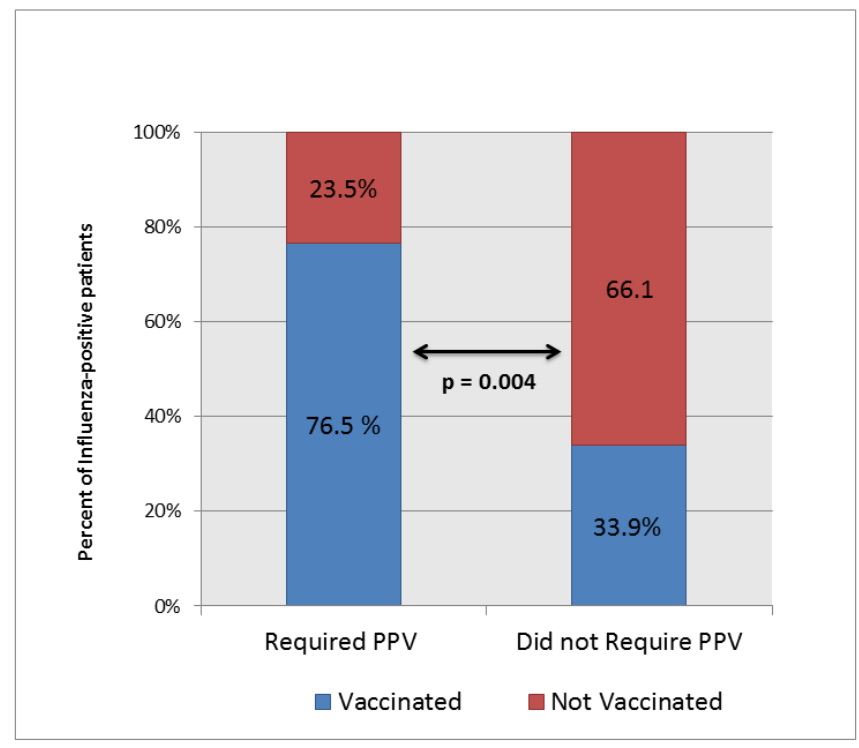

Fig. (2). $100 \%$ Stacked Column chart showing high rate of vaccination in patients requiring positive pressure ventilation (PPV) as compared to those who did not require PPV.

$(p=0.275)$ (Fig. 4). Similarly, the prevalence of COPD was not statistically predictive of ICU admission $(\mathrm{p}=0.902)$ or of need for PPV ( $\mathrm{p}=0.486$ ) (Figs. 5, 6).

\section{Mortality}

A total of four people (6\%) died due to Influenza-related complications. (Table 1) All had at least one significant underlying co-morbid condition (COPD, CHF, DM). The median age of patients who died was 65 years (range 64-66). Of these, 2 were immunocompromised; one had been receiving chemotherapy for metastatic cancer and the other was on high-dose steroids. Three out of the 4 had been vaccinated, 3 had influenza $\mathrm{A}$, and $\mathrm{H} 1 \mathrm{~N} 1$ was documented in 2 of those 3. All 4 patients had received antibiotics for pneumonia/sepsis and 3/4 had received oseltamivir.

\section{DISCUSSION}

For most individuals the flu is self-limiting and recovery occurs in less than two weeks even without medical care or antiviral drugs $[1,2]$. Some subsets of the population are at higher risk for more severe disease. The elderly ( $\geq 65$ years of age) appear to have the highest risk of increased morbidity including respiratory failure, and mortality; it is estimated that over $60 \%$ of all seasonal-influenza-related hospitalizations and $90 \%$ of seasonal-influenza-related deaths in the United States each year occur in elderly persons [2, 3-7]. The CDC states that flu vaccination can reduce flu illnesses, doctors' visits, and missed work and school due to flu as well as prevent flu-related hospitalizations and deaths, especially in the elderly with underlying medical conditions [4]. The $\mathrm{CDC}$ recommends annual flu vaccination for all individuals $>6$ months of age [4].

To the best of our knowledge, this is the first study in the United States describing influenza morbidity and mortality in this high-risk group admitted to a tertiary care center during 2013-2014 flu season. Although, it is a protocol at CAVHS to test all patients with flu-like symptoms with rapid antigen testing and/or RT-PCR, we presume that all or most patients admitted to CAVHS during the 2013-2014 flu season, were captured. If any were missed, there is no way in which the methods used in this study would have caused a collection bias. The study group represents the older population at most risk for morbidity and death according to the CDC; only one patient was under 50 years of age, with the majority over 60 years of age and a mean age of 66 . The vast majority also had co-morbidities. Of note, this study of a VA population included only three women; if women have a different profile of influenzal illness, those differences were not captured by this study.

When the influenza-infected patients were divided according to vaccination status, there were salient differences; frequency of ICU admission was approximately twice as high for vaccinated patients, and vaccinated patients were more than twice as likely to receive mechanical ventilatory support for respiratory failure. Our data thus suggest that vaccination offered no protection against severe illness in this population of patients admitted to the hospital. The lack of protection documented by this study occurred despite the facts that 1) the predominant influenza A strain $(80 \%)$ in our study population was $\mathrm{pH} 1 \mathrm{~N} 1$, consistent with the recent CDC interim report (8) and 2) this season's vaccine was considered to have provided substantial protection against the circulating strains [8]. There was also no protection against mortality; three out of 4 patients in this study who died had received influenza vaccine.

The most common underlying medical condition in this cohort of elderly patients was COPD (36/70, 51\%). COPD has been described previously as one of the major comorbidities in hospitalized influenza patients requiring ICU admission and/or PPV for respiratory failure [9]. In our study patients, the presence of COPD was not a significant predictor either of ICU admission or of the need for mechanical ventilation (Figs. 5, 6). Similarly, there was no significant correlation between age and ICU admission or need for ventilatory support for respiratory failure. The 


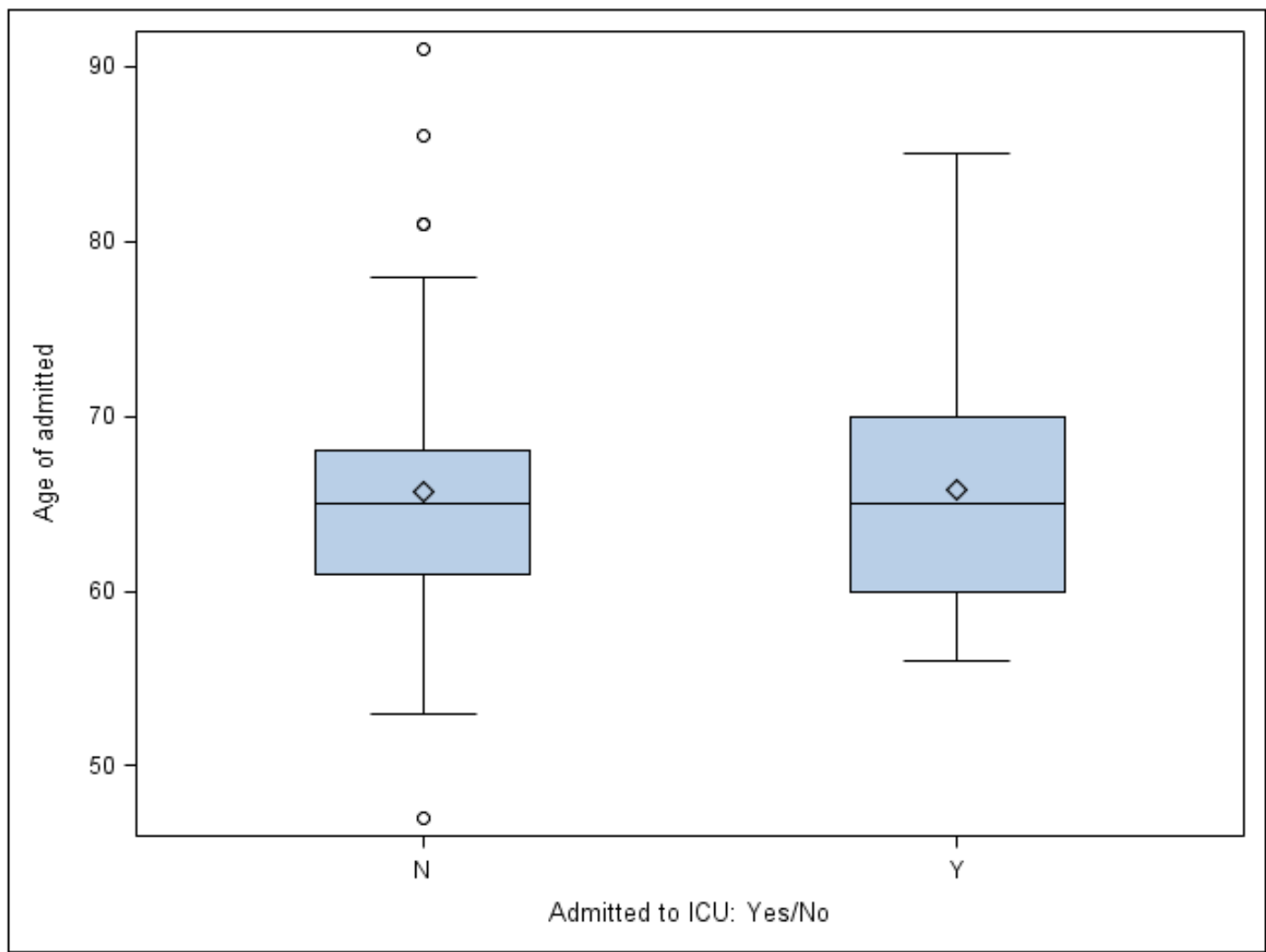

Fig. (3). Box-and-whisker plots comparing the mean age (represented by diamond within the box) in patients who were admitted to ICU (Y) and in those treated in non-ICU setting $(\mathrm{N})$. There was no significant difference in the mean levels among the two groups $(\mathrm{P}=0.969)$. The horizontal line within the box represents the median and circles represent the outliers.

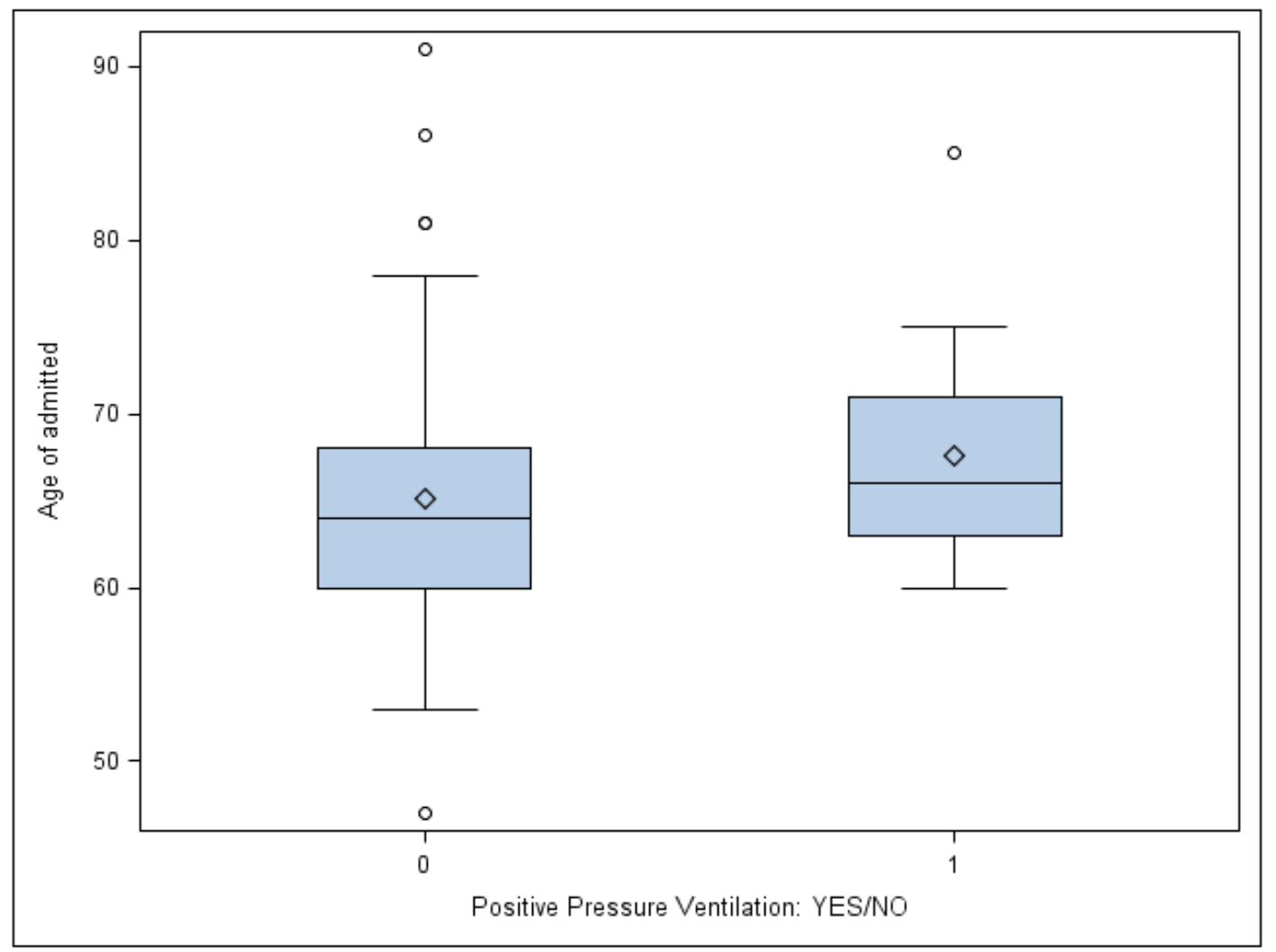

Fig. (4). Box-and-whisker plots comparing the mean age (represented by diamond) in patients who required positive pressure ventilation (labeled as 1) as compared to those who did not require PPV (labeled as 0). There was no significant difference in the mean levels among the two groups $(\mathrm{p}=0.275)$. 


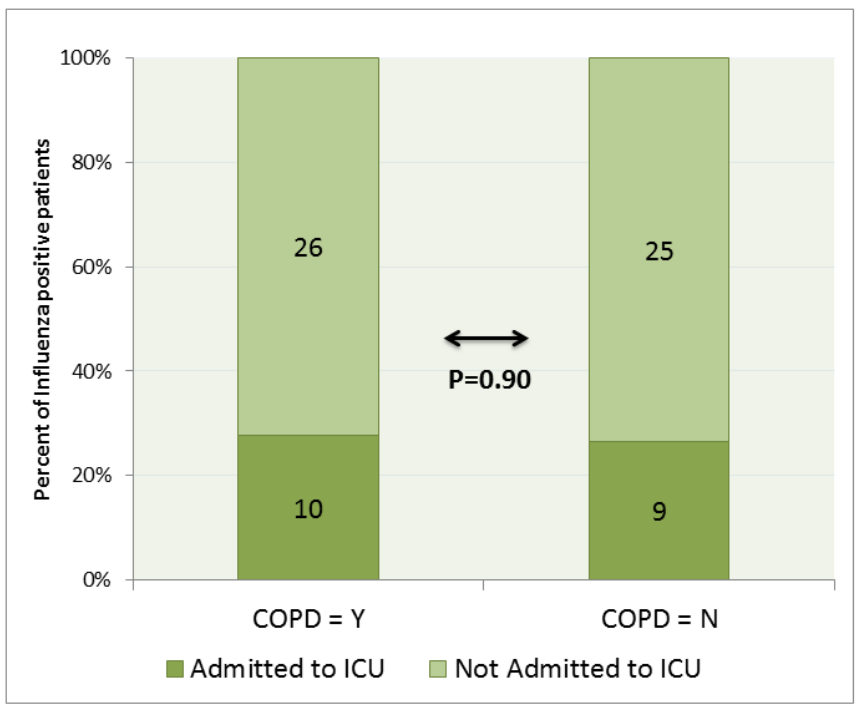

Fig. (5). $100 \%$ Stacked Column chart comparing the prevalence of COPD among those who required ICU care $v s$ no ICU $(\mathrm{p}=0.902)$.

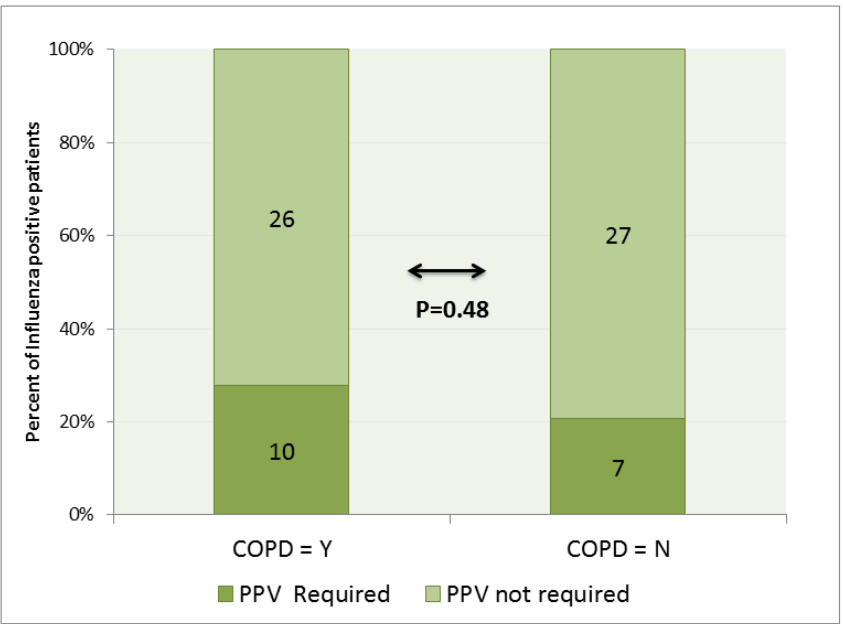

Fig. (6). $100 \%$ Stacked Column chart comparing the prevalence of COPD among those who required who required positive pressure ventilation (PPV) as compared to those who did not require PPV (P $=0.486$ ).

findings thus suggest that the likelihoods of ICU admission and/or PPV requirement were related to the severity of influenza illness itself rather than to COPD or age.

These findings are consistent with published studies that suggest minimal or no influenza vaccine effectiveness in preventing mortality in elderly people admitted with influenza [5, 6, 10-13]. In a multivariate analysis of patients hospitalized with influenza during the 2009 season, the pH1N1 Virus Hospitalizations Investigation Team showed no overall mortality benefit from prior influenza vaccination [9]. Early antiviral treatment initiation (within 2 days of illness) was the only variable significantly associated with a positive outcome [9]. Another large study which included 1.4 million residents over the course of 15 influenza seasons concluded that influenza vaccination prevented only about $4 \%$ of influenza-associated hospitalizations and deaths occurring after hospitalizations among older adults in Ontario, Canada [10]. Some studies have concluded the contrary, that influenza vaccination reduces all-cause winter- season mortality in the elderly by $50 \%[14,15]$. Simonsen and colleagues clearly demonstrated that this magnitude of mortality benefit is not possible and irrefutably showed that reported high vaccine effectiveness was due to flawed designs and selection biases in those studies [16].

Apart from mortality, the broader efficacy of the influenza vaccine has come under question [16-21]. The estimates of efficacy listed in the interim CDC report are limited to the prevention of outpatient medical visits [8]. Furthermore, the CDC itself recommends further studies to measure vaccine effectiveness against more severe illness outcomes such as hospitalization and death [8].

The limitations of this study include the following: 1) It is an observational single-center study with a limited number of patients. 2) Only one influenza season was covered. 3) As noted, because of the veteran population, the study group consisted almost exclusively of men, and the findings cannot be confidently applied to women. There are several strengths: 1) Routine in-house PCR testing for influenza implies that most if not all patients admitted with influenza were captured. 2) No patient's vaccination status was based upon self-reporting. Most veterans use the VA hospital exclusively for all care, and any vaccinations (or refusals) are well documented and easily found in the electronic health records. 3) This study was conducted during a season in which seasonal influenza vaccine was considered adequate, providing substantial protection against the year's circulating strains, including $\mathrm{pH} 1 \mathrm{~N} 1$. Since vaccine effectiveness may vary year-to-year depending on the circulating virus strain and the vaccine match, our results and conclusions seem to be clinically more relevant during this well-matched season.

Based on the current literature and these results, further vaccine effectiveness studies to assess vaccine benefits in preventing serious complications of influenza in the elderly are clearly needed. The best approach would be to perform large randomized placebo-controlled trials. However this is not feasible at present and is an ethically arduous proposition, especially given current influenza vaccination policies. We would also suggest that it is time for the CDC and other institutions to re-evaluate blanket policies with respect to vaccination and to pave the way for randomized trials.

In summary, this study of the impact of prior influenza vaccine on severity of illness in older patients admitted with the flu found no evidence of a protective benefit from the vaccine. In fact, the prevalence of severe disease and respiratory failure was statistically higher in the vaccinated patients. These data add to a growing body of literature that raises questions about the overall efficacy of the vaccine. While even more data are awaited, we suggest a high index of suspicion in people presenting with flu-like illness, protocol-based testing to ensure rapid diagnosis, and early treatment.

\section{AUTHORS CONTRIBUTION}

All authors made a contribution in the content and writing of the manuscript including final revision of the manuscript for important intellectual content. MJ was the principal investigator and contributed the original idea for the study. DC and PM collected the data. DC, PM, TB, and 
MJ had input into the study design. DC, PM, and MJ performed data analysis and interpretation.

\section{CONFLICT OF INTEREST}

The authors confirm that this article content has no conflict of interest.

\section{ACKNOWLEDGEMENTS}

We thank Dr. Anita Joshi, BDS, MPH for critical review of the statistical section of the manuscript.

\section{REFERENCES}

[1] Thompson WW, Shay DK, Weintraub E, et al. Mortality associated with influenza and respiratory syncytial virus in the United States. JAMA 2003; 289: 179-86.

[2] Centers for Disease Control and Prevention. [Accessed 2014, April 15] Available from: http://www.cdc.gov/flu/about/disease/high_ri sk.htm

[3] Thompson WW, Shay DK, Weintraub E, et al. Influenza-associated hospitalizations in the United States. JAMA 2004; 292: 1333-40.

[4] Centers for Disease Control and Prevention [Accessed 2014, April 15]. Available from: http://www.cdc.gov/flu/pdf/freeresources/gene ral/flu-vaccine-benefits.pdf

[5] Rivetti D, Jefferson $\mathrm{T}$, Thomas $\mathrm{R}$, et al. Vaccines for preventing influenza in the elderly. Cochrane Database Syst Rev 2006; 3: CD004876.

[6] Osterholm MT, Kelley NS, Sommer A, Belongia EA. Efficacy and effectiveness of influenza vaccines: A systematic review and metaanalysis. Lancet Infect Dis 2012; 12: 36-44.

[7] Centers for Disease Control and Prevention [Accessed 2014, April 15]. Available from: http://www.cdc.gov/flu/about/disease/65over. htm

[8] Flannery B, Thaker SN, Clippard J, et al. Centers for disease control and prevention. interim estimates of 2013-14 seasonal influenza vaccine effectiveness - United States, February 2014. MMWR Morb Mortal Wkly Rep 2014; 63(7): 137-42.

[9] Jain S, Kamimoto L, Bramley AM, et al. Pandemic influenza A (H1N1) virus hospitalizations investigation team: Hospitalized patients with 2009 H1N1 influenza in the United States, April-June 2009. N Engl J Med 2009; 361: 1935-44.

[10] Ridenhour BJ, Campitelli MA, Kwong JC, et al. Effectiveness of inactivated influenza vaccines in preventing influenza-associated deaths and hospitalizations among ontario residents aged $\$ 65$ years: Estimates with generalized linear models accounting for healthy vaccinee effects. PLoS ONE 2013; 8(10): e76318.

[11] Simonsen L, Reichert TA, Viboud C, Blackwelder WC, Taylor RJ, Miller MA. Impact of influenza vaccination on seasonal mortality in the US elderly population. Arch Intern Med 2005; 165: 265-72.

[12] Rizzo C, Viboud C, Montomoli E, Simonsen L, Miller MA. Influenza-related mortality in the Italian elderly: No decline associated with increasing vaccination coverage. Vaccine 2006; 24 : 6468-75.

[13] Jefferson T, Rivetti D, Rivetti A, Rudin M, Di Pietrantonj C, Demichelli V. Efficacy and effectiveness of influenza vaccine in elderly people: A systematic review. Lancet 2005; 366: 1165-74.

[14] Vu T, Farish S, Jenkins M, Kelly H. A meta-analysis of effectiveness of influenza vaccine in persons aged 65 years and over living in the community. Vaccine 2002; 20: 1831-6.

[15] Gross PA, Hermogenes AW, Sacks HS, Lau J, Levandowski RA. The efficacy of influenza vaccine in elderly persons. A metaanalysis and review of the literature. Ann Intern Med 1995; 123 518-27.

[16] Simonsen L, Taylor RJ, Viboud C, Miller MA, A Jackson LA. Mortality benefits of influenza vaccination in elderly people: An ongoing controversy. Lancet Infect Dis 2007; 7: 658-66

[17] Baxter R, Lee J, Fireman B. Evidence of bias in studies of influenza vaccine effectiveness in elderly patients. J Infect Dis 2010; 201: 186-9.

[18] Jackson LA, Nelson JC, Benson P, et al. Functional status is a confounder of the association of influenza vaccine and risk of all cause mortality in seniors. Int J Epidemiol 2006; 35: 345-52.

[19] Bawazeer GA, Jefferson T, Al-Ansary LA, Ferroni E, Rivetti A, Di Pietrantonj C. Vaccines for preventing influenza in healthy adults. Cochrane Database Syst Rev 2014; 3: D001269.

[20] Wong K, Campitelli MA, Stukel TA, Kwong JC. Estimating influenza vaccine effectiveness in community-dwelling elderly patients using the instrumental variable analysis method. Arch Intern Med 2012; 172: 484-91.

[21] Ferdinands JM, Gargiullo P, Haber M, Moore M, Belongia EA, Shay DK. Inactivated influenza vaccines for prevention of community-acquired pneumonia: the limits of using nonspecific outcomes in vaccine effectiveness studies. Epidemiology 2013; 24: $530-7$. 